\title{
Optimal bounded control of first-passage failure of strongly non-linear oscillators under combined harmonic and white-noise excitations
}

\author{
W.Q. Zhu ${ }^{\mathrm{a}, \mathrm{b}, *}$, Y.J. Wu ${ }^{\mathrm{a}}$ \\ ${ }^{a}$ Department of Mechanics, Zhejiang University, Hangzhou 310027, People's Republic of China \\ ${ }^{\mathrm{b}}$ State Key Laboratory of Nonlinear Mechanics, Institute of Mechanics, Chinese Academy of Sciences, \\ Beijing 100008, People's Republic of China
}

Received 10 April 2002; accepted 16 February 2003

\begin{abstract}
A procedure for designing the optimal bounded control of strongly non-linear oscillators under combined harmonic and white-noise excitations for minimizing their first-passage failure is proposed. First, a stochastic averaging method for strongly non-linear oscillators under combined harmonic and whitenoise excitations using generalized harmonic functions is introduced. Then, the dynamical programming equations and their boundary and final time conditions for the control problems of maximizing reliability and of maximizing mean first-passage time are formulated from the averaged Ito equations by using the dynamical programming principle. The optimal control law is derived from the dynamical programming equations and control constraint. Finally, the conditional reliability function, the conditional probability density and mean of the first-passage time of the optimally controlled system are obtained from solving the backward Kolmogorov equation and Pontryagin equation. An example is given to illustrate the proposed procedure and the results obtained are verified by using those from digital simulation.
\end{abstract}

(C) 2003 Elsevier Ltd. All rights reserved.

\section{Introduction}

Excitations of dynamical systems are usually classified as deterministic and random ones. However, many mechanical and structural systems are subject to both harmonic and random excitations. A typical example of such systems is helicopter rotor blade vibration during forward flight in a turbulent atmosphere. The random part of the combined excitations is usually modelled as Gaussian white-noise or wideband random process. A set of combined harmonic and random

\footnotetext{
*Corresponding author. Tel.: 86-571-87991150; fax: 86-571-87952651.

E-mail address: wqzhu@yahoo.com (W.Q. Zhu).
} 
excitations is then equivalent to a narrowband random excitation. A dynamical system subject to combined harmonic and random excitations is often analyzed by using the method of multiple scales or the method of stochastic averaging. For example, a weakly non-linear system under both external harmonic and white-noise excitations was investigated by Nayfeh and Serhan [1] using the method of multiple scales. The stability of linear systems under combined parametric harmonic and random excitations and the response of non-linearly damped systems under combined external and (or) parametric harmonic and random excitations have been studied by several scholars [2-8] using the classical method of stochastic averaging. To study the response of oscillators with strongly non-linear stiffness under combined harmonic and white-noise excitations, a method of stochastic averaging was proposed recently by the present first author and his co-worker [9] using the so-called generalized harmonic functions [10] and the stochastic jump and its bifurcation of Duffing oscillator with hardening spring subject to combined harmonic and white-noise excitations were studied by using this stochastic averaging method.

In the past two decades, substantial advances have been made in the field of structural vibration control [11]. In most studies, structures are modelled as linear systems and feedback control is also linear. The active control of strongly non-linear systems is comparatively much less studied. The optimal polynomial control of autonomous Duffing oscillator was studied by Suhardjo et al. [12] and Agrawal et al. [13]. The active control of Duffing oscillator under harmonic excitation has been investigated by Abdel-Rohman and Nayfeh [14] for reducing the response, by Chow and Maestrello [15] for stabilizing the system, and by Ji [16] for removing or delaying the jump and hysteresis in the response. In these studies, a control force with undetermined parameters is first assumed and then the parameters are determined by analyzing the response, stability or bifurcation of the controlled system. Thus, these controllers are not optimal in rigorous sense. On the other hand, the optimal control of Duffing oscillator under Gaussian white-noise excitations was studied by Yoshida [17] using statistical linearization, by Crespo and Sun [18] using generalized cell mapping with short-time Gaussian approximation and by Zhu et al. [19] using stochastic averaging method. To the authors' knowledge, so far the optimal control of strongly non-linear systems under combined harmonic and random excitations has not been investigated.

The general purpose of structural vibration control is to keep the state of controlled structures within a suitable domain from the point view of performance or safety. For the structures under random dynamical loading, first-passage failure is a major failure mode. Therefore, the study of feedback minimization of first-passage failure of randomly excited structural systems is of great significance. However, such a study has been made only for quasi-non-integrable Hamiltonian systems [20]. For strongly non-linear systems under combined harmonic and random excitation, no such work has been done.

In the present paper, the optimal bounded control of strongly non-linear oscillators under combined harmonic and white-noise excitations for minimizing the first-passage failure is studied. The motion equation of such a system is first reduced to averaged Itô equations by using the stochastic averaging method [9]. Then, the dynamical programming equations for the control problems of maximizing reliability and of maximizing mean first-passage time and their boundary and final time conditions are formulated from the averaged Itô equations by applying the dynamical programming principle [21]. The optimal control law is determined from the dynamical programming equations and control constraint. Finally, the conditional reliability function, the probability density and mean of first-passage time of optimally controlled system are obtained 
from solving the backward Kolmogorov equation and Pontryagin equation associated with completely averaged system. A Duffing oscillator under external harmonic excitation and external and parametric white-noise excitations is taken as an example to illustrate the proposed procedure and the effectiveness of the control on the first-passage failure.

\section{Stochastic averaging}

Consider a weakly controlled strongly non-linear oscillator subject to light linear and (or) nonlinear damping and weak external and (or) parametric excitations of harmonic function and white noises. The equation of motion of the system is of the form

$$
\ddot{X}+g(X)=\varepsilon f(X, \dot{X}, \Omega t)+\varepsilon u(X, \dot{X})+\varepsilon^{1 / 2} h_{k}(X, \dot{X}) \xi_{k}(t), \quad k=1,2, \ldots, m,
$$

where $\varepsilon$ is a small parameter; $\varepsilon f$ denotes light damping and weak external and (or) parametric harmonic excitation with frequency $\Omega ; \varepsilon^{1 / 2} h_{k} \xi_{k}(t)$ represent weak external and (or) parametric white-noise excitations; $\xi_{k}(t)$ are Gaussian white-noises in the sense of Stratonovich with correlation functions $E\left[\xi_{k}(t) \xi_{l}(t+\tau)\right]=2 D_{k l} \delta(\tau)$; and $\varepsilon u$ denotes weakly feedback control force.

When $\varepsilon=0$, system (1) degenerates to a non-linear conservative oscillator

$$
\ddot{x}+g(x)=0 .
$$

The Hamiltonian (total energy) of the oscillator is

$$
H=\frac{1}{2} \dot{x}^{2}+U(x)
$$

where

$$
U(x)=\int_{0}^{x} g(u) \mathrm{d} u
$$

is the potential energy. Assume that oscillator (2) has a family of periodic solutions surrounding equilibrium $(b, 0)$ in phase plane $(x, \dot{x})$. The periodic solution can be expressed as

$$
\begin{gathered}
x(t)=a \cos \varphi(t)+b, \\
\dot{x}(t)=-a v(a, \varphi) \sin \varphi(t),
\end{gathered}
$$

where

$$
\begin{gathered}
\varphi(t)=\tau(t)+\theta, \\
v(a, \varphi)=\frac{\mathrm{d} \tau}{\mathrm{d} t}=\sqrt{\frac{2[U(a+b)-U(a \cos \varphi+b)]}{a^{2} \sin ^{2} \varphi},}
\end{gathered}
$$

$a$ and $b$ are constants and related to $H$ as follows:

$$
U(a+b)=U(-a+b)=H .
$$

$\cos \varphi(t)$ and $\sin \varphi(t)$ are called generalized harmonic functions [10]. Obviously, $a$ and $v(a, \varphi)$ are the amplitude and instantaneous frequency of the oscillator, respectively, and $\theta$ is the phase angle of the response relative to harmonic excitation. Expanding $v^{-1}(a, \varphi)$ in Eq. (8) 
into Fourier series

$$
v^{-1}(a, \varphi)=C_{0}(a)+\sum_{n=1}^{\infty} C_{n}(a) \cos n \varphi,
$$

and then integrating Eq. (10) with respect to $\tau$ yield

$$
t=C_{0}(a) \tau+\sum_{n=1}^{\infty} \frac{1}{n} C_{n}(a) \sin n \varphi .
$$

Letting $\tau=2 \pi$ leads to average period

$$
T(a)=2 \pi C_{0}(a)
$$

and average frequency

$$
\omega(a)=\frac{1}{C_{0}(a)}
$$

Thus, the following approximate expression will be used in the averaging:

$$
\varphi(t) \approx \omega(a) t+\theta
$$

When $\varepsilon$ is very small, the solution to system (1) can be assumed of the following form:

$$
X(t)=A \cos \Phi(t)+B, \quad \dot{X}(t)=-A v(A, \Phi) \sin \Phi(t),
$$

where

$$
\begin{gathered}
\Phi(t)=\tau(t)+\Theta(t), \\
v(A, \Phi)=\frac{\mathrm{d} \tau}{\mathrm{d} t}=\sqrt{\frac{2[U(A+B)-U(A \cos \Phi+B)]}{A^{2} \sin ^{2} \Phi}},
\end{gathered}
$$

and $A, \Phi, \tau$ and $v$ are all random processes. Treating Eq. (15) as generalized van der Pol transformation from $X, \dot{X}$ to $A, \Theta$, one can obtain the following equations for $A$ and $\Theta$ :

$$
\begin{aligned}
& \frac{\mathrm{d} A}{\mathrm{~d} t}=\varepsilon F_{1}^{(1)}(A, \Phi, \Omega t)+\varepsilon F_{1}^{(2)}(A, \Phi, u)+\varepsilon^{1 / 2} H_{1 k}(A, \Phi) \xi_{k}(t), \\
& \frac{\mathrm{d} \Theta}{\mathrm{d} t}=\varepsilon F_{2}^{(1)}(A, \Phi, \Omega t)+\varepsilon F_{2}^{(2)}(A, \Phi, u)+\varepsilon^{1 / 2} H_{2 k}(A, \Phi) \xi_{k}(t),
\end{aligned}
$$


where

$$
\begin{aligned}
& F_{1}^{(1)}=\frac{-A}{g(A+B)(1+h)} f(A \cos \Phi+B,-A v(A, \Phi) \sin \Phi, \Omega t) v(A, \Phi) \sin \Phi, \\
& F_{1}^{(2)}=\frac{-A u}{g(A+B)(1+h)} v(A, \Phi) \sin \Phi, \\
& F_{2}^{(1)}=\frac{-1}{g(A+B)(1+h)} f(A \cos \Phi+B,-A v(A, \Phi) \sin \Phi, \Omega t) v(A, \Phi)(\cos \Phi+h), \\
& F_{2}^{(2)}=\frac{-u}{g(A+B)(1+h)} v(A, \Phi)(\cos \Phi+h), \\
& H_{1 k}=\frac{-A}{g(A+B)(1+h)} h_{k}(A \cos \Phi+B,-A v(A, \Phi) \sin \Phi) v(A, \Phi) \sin \Phi, \\
& H_{2 k}=\frac{-1}{g(A+B)(1+h)} h_{k}(A \cos \Phi+B,-A v(A, \Phi) \sin \Phi) v(A, \Phi)(\cos \Phi+h), \\
& h=\frac{\mathrm{d} B}{\mathrm{~d} A}=\frac{g(-A+B)+g(A+B)}{g(-A+B)-g(A+B)} .
\end{aligned}
$$

Eq. (18) can be modelled as Stratonovich stochastic differential equations and then converted into Itô stochastic differential equations by adding Wong-Zakai correction terms. The result is

$$
\begin{aligned}
\mathrm{d} A & =\varepsilon\left[m_{1}^{(1)}(A, \Phi, \Omega t)+F_{1}^{(2)}(A, \Phi, u)\right] \mathrm{d} t+\varepsilon^{1 / 2} \sigma_{1 r}(A, \Phi) \mathrm{d} B_{r}(t), \\
\mathrm{d} \Theta & =\varepsilon\left[m_{2}^{(1)}(A, \Phi, \Omega t)+F_{2}^{(2)}(A, \Phi, u)\right] \mathrm{d} t+\varepsilon^{1 / 2} \sigma_{2 r}(A, \Phi) \mathrm{d} B_{r}(t), \quad r=1,2, \ldots, m,
\end{aligned}
$$

where $B_{r}(t)$ are independent unit Wiener processes,

$$
\begin{aligned}
& m_{i}^{(1)}=F_{i}^{(1)}+D_{k l} \frac{\partial H_{i k}}{\partial A} H_{1 l}+D_{k l} \frac{\partial H_{i k}}{\partial \Phi} H_{2 l}, \\
& b_{i j}=\sigma_{i r} \sigma_{j r}=2 D_{k l} H_{i k} H_{j l}, \quad i, j=1,2, \quad k, l, r=1,2, \ldots, m .
\end{aligned}
$$

In non-resonant case, the harmonic excitation has no effect on the first approximation of the response. So, only resonant case is considered in the following. Assume that we are interested in resonant case, i.e.,

$$
\frac{\Omega}{\omega(A)}=\frac{q}{p}+\varepsilon \sigma
$$

where $p$ and $q$ are relatively prime positive small integers and $\varepsilon \sigma$ is the detuning parameter. In this case, multiplying Eq. (11) by $\Omega$ yields

$$
\Omega t=\frac{q}{p} \Phi+\varepsilon \sigma \tau-\frac{q}{p} \Theta+\Omega \sum_{n=1}^{\infty} \frac{1}{n} C_{n}(A) \sin n \Phi .
$$

Introduce new variable $\Gamma$ such that

$$
\Gamma=\varepsilon \sigma \tau-\frac{q}{p} \Theta
$$


which denotes the phase difference of the response and harmonic excitation. Then, Eq. (23) can be rewritten as

$$
\Omega t=\Psi+\Gamma,
$$

where

$$
\Psi=\Psi(A, \Phi)=\frac{q}{p} \Phi+\Omega \sum_{n=1}^{\infty} \frac{1}{n} C_{n}(A) \sin n \Phi .
$$

Eq. (20) can be transformed into

$$
\begin{aligned}
\mathrm{d} A=\varepsilon\left[m_{1}^{(1)}(A, \Phi, \Psi+\Gamma)+F_{1}^{(2)}(A, \Phi, u)\right] \mathrm{d} t+\varepsilon^{1 / 2} \sigma_{1 r}(A, \Phi) \mathrm{d} B_{r}(t), \\
\mathrm{d} \Gamma=\left\{\varepsilon\left[m_{2}^{(1)}(A, \Phi, \Psi+\Gamma)+F_{2}^{(2)}(A, \Phi, u)\right]\left(-\frac{q}{p}\right)+\left(\frac{\Omega}{\omega(A)}-\frac{q}{p}\right) v(A, \Phi)\right\} \mathrm{d} t \\
-\varepsilon^{1 / 2} \frac{q}{p} \sigma_{2 r}(A, \Phi) \mathrm{d} B_{r}(t) .
\end{aligned}
$$

$A$ and $\Gamma$ are slowly varying processes while $\Phi$ is rapidly varying process. Averaging the drift and diffusion coefficients with respect to $\Phi$ yields the following averaged Itô equations:

$$
\begin{aligned}
\mathrm{d} \bar{A} & =\varepsilon\left[\bar{m}_{1}^{(1)}(\bar{A}, \bar{\Gamma})+\left\langle F_{1}^{(2)}(A, \Phi, u)\right\rangle_{\Phi}\right] \mathrm{d} t+\varepsilon^{1 / 2} \bar{\sigma}_{1 r}(\bar{A}) \mathrm{d} B_{r}(t), \\
\mathrm{d} \bar{\Gamma} & =\varepsilon\left[\bar{m}_{2}^{(1)}(\bar{A}, \bar{\Gamma})-\frac{q}{p}\left\langle F_{2}^{(2)}(A, \Phi, u)\right\rangle_{\Phi}\right] \mathrm{d} t+\varepsilon^{1 / 2} \bar{\sigma}_{2 r}(\bar{A}) \mathrm{d} B_{r}(t),
\end{aligned}
$$

where

$$
\begin{aligned}
& \bar{m}_{1}^{(1)}=\left\langle m_{1}^{(1)}(A, \Phi, \Psi+\Gamma)\right\rangle_{\Phi}, \\
& \bar{m}_{2}^{(1)}=\left\langle m_{2}^{(1)}(A, \Phi, \Psi+\Gamma)\left(-\frac{q}{p}\right)+\frac{v(A, \Phi)}{\varepsilon}\left(\frac{\Omega}{\omega(A)}-\frac{q}{p}\right)\right\rangle_{\Phi}, \\
& \bar{b}_{11}=\bar{\sigma}_{1 r} \bar{\sigma}_{1 r}=\left\langle\sigma_{1 r} \sigma_{1 r}\right\rangle_{\Phi^{u}} \bar{b}_{22}=\bar{\sigma}_{2 r} \bar{\sigma}_{2 r}=\frac{q^{2}}{p^{2}}\left\langle\sigma_{2 r} \sigma_{2 r}\right\rangle_{\Phi}, \\
& \bar{b}_{12}=\bar{b}_{21}=\bar{\sigma}_{1 r} \bar{\sigma}_{2 r}=-\frac{q}{p}\left\langle\sigma_{1 r} \sigma_{2 r}\right\rangle_{\Phi},
\end{aligned}
$$

and $\langle\cdot\rangle_{\Phi}$ denotes the averaging with respect to $\Phi$ from 0 to $2 \pi$.

Amplitude $A$ of displacement $X$ is related to Hamiltonian $H$ by Eq. (9). Eq. (28) can be converted into the following Itô equations for $\bar{H}$ and $\bar{\Gamma}$ by using Itô differential rule:

$$
\begin{aligned}
\mathrm{d} \bar{H}= & \varepsilon\left\{\frac{\mathrm{d} U}{\mathrm{~d} \bar{A}}\left[\bar{m}_{1}^{(1)}(\bar{A}, \bar{\Gamma})+\left\langle F_{1}^{(2)}(A, \Phi, u)\right\rangle_{\Phi}\right]+\frac{1}{2} \frac{\mathrm{d}^{2} U}{\mathrm{~d} \bar{A}^{2}} \bar{\sigma}_{1 r} \bar{\sigma}_{1 r}\right\} \mathrm{d} t \\
& +\varepsilon^{1 / 2} \frac{\mathrm{d} U}{\mathrm{~d} \bar{A}} \bar{\sigma}_{1 r} \mathrm{~d} B_{r}(t), \\
\mathrm{d} \bar{\Gamma}= & \varepsilon\left[\bar{m}_{2}^{(1)}(\bar{A}, \bar{\Gamma})-\frac{q}{p}\left\langle F_{2}^{(2)}(A, \Phi, u)\right\rangle_{\Phi}\right] \mathrm{d} t+\varepsilon^{1 / 2} \bar{\sigma}_{2 r} \mathrm{~d} B_{r}(t),
\end{aligned}
$$

where $\mathrm{d} U / \mathrm{d} \bar{A}=g(\bar{A})(1+h), \mathrm{d}^{2} U / \mathrm{d} \bar{A}^{2}=\mathrm{d}[g(\bar{A})(1+h)] / \mathrm{d} \bar{A}, \bar{A}$ is replaced by $\bar{H}$ using relation $\bar{A}=U^{-1}(\bar{H})-B$.

Note that $X, \dot{X}$ in Eq. (1) is non-homogeneous diffusion Markov process while $\bar{A}, \bar{\Gamma}$ in Eq. (28) and $\bar{H}, \bar{\Gamma}$ in Eq. (30) are homogeneous diffusion Markov processes. In the following Eq. (28) or 
(30) rather than Eq. (1) will be used to design the optimal bounded control for minimizing the first-passage failure of system (1).

\section{Dynamical programming equations}

$A$ is the amplitude of displacement, $H$ is the total energy of the system, and $\Gamma$ is the phase difference of the response and harmonic excitation. The limiting state of system may specified in terms of $A$ or $H$. Since $A$ and $H$ are related by Eq. (9) and both $A$ and $H$ are non-negative, it is equivalent to specify $A$ or $H$ for the limiting state. It is reasonable to assume that the first-passage failure occurs once averaged amplitude $\bar{A}(t)$ exceed critical value $\bar{A}_{c}$ for the first time. In phase plane $(\bar{a}, \bar{\gamma})$, the safety domain $\Omega_{s}$ is inside of the two parallel lines $\bar{a}=0$ and $\bar{a}_{c}$ (see Fig. 1). For the control problem of maximizing reliability, introduce value function

$$
V(t, \bar{a}, \bar{\gamma})=\sup _{u \in U_{c}} P\left\{[\bar{A}(\tau, u), \bar{\Gamma}(\tau, u)] \in \Omega_{s}, \quad \tau \in\left[t, t_{f}\right]\right\},
$$

where $u \in U_{c}$ denotes the control constraint. Eq. (31) implies that $V(t, \bar{a}, \bar{\gamma})$ is the reliability function of optimally controlled system (28). Based on the stochastic dynamical programming principle [21], the following dynamical programming equation can be derived:

$$
\begin{gathered}
\sup _{u \in U} \varepsilon\left\{\frac{\partial}{\partial t}+\left[\bar{m}_{1}^{(1)}+\left\langle F_{1}^{(2)}\right\rangle_{\varphi}\right] \frac{\partial}{\partial \bar{a}}+\left[\bar{m}_{2}^{(1)}-\frac{q}{p}\left\langle F_{2}^{(2)}\right\rangle_{\varphi}\right] \frac{\partial}{\partial \bar{\gamma}}+\frac{1}{2} \bar{b}_{11} \frac{\partial^{2}}{\partial \bar{a}^{2}}\right. \\
\left.\quad+\frac{1}{2} \bar{b}_{22} \frac{\partial^{2}}{\partial \bar{\gamma}^{2}}+\bar{b}_{12} \frac{\partial^{2}}{\partial \bar{a} \partial \bar{\gamma}}\right\} V(t, \bar{a}, \bar{\gamma})=0, \quad \bar{a}, \bar{\gamma} \in \Omega_{s}, \quad t \in\left[0, t_{f}\right],
\end{gathered}
$$

where $\bar{b}_{i j}=\bar{\sigma}_{i r} \bar{\sigma}_{j r}$. The boundary conditions associated with Eq. (32) are

$$
\begin{gathered}
V\left(t, \bar{a}_{c}, \bar{\gamma}\right)=0, \\
V(t, 0, \bar{\gamma})=\text { finite, }
\end{gathered}
$$

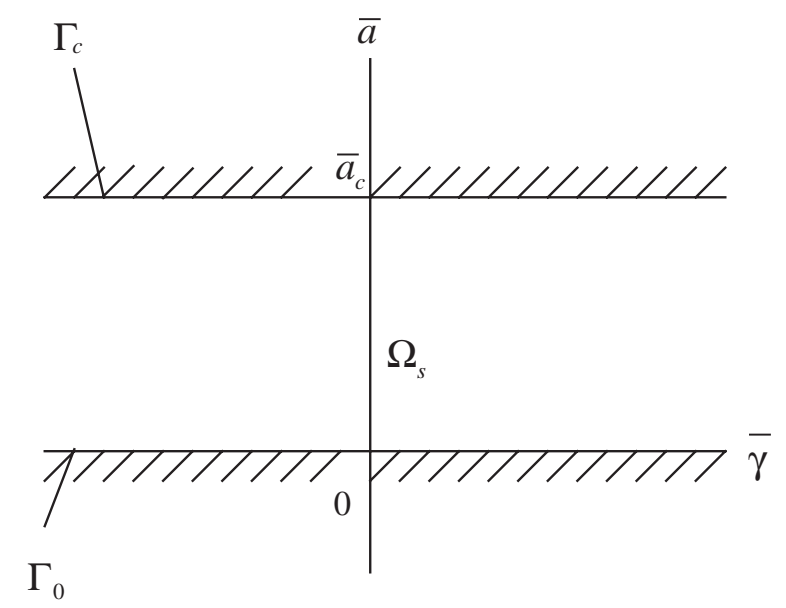

Fig. 1. Safety domain $\Omega_{s}$ and its boundaries on plane $(\bar{a}, \bar{\gamma})$ for system (66). 


$$
V(t, \bar{a}, \bar{\gamma}+2 n \pi)=V(t, \bar{a}, \bar{\gamma})
$$

and the final time condition is

$$
V\left(t_{f}, \bar{a}, \bar{\gamma}\right)=1, \quad \bar{a} \in \Omega_{s} .
$$

Eqs. (32)-(35) are the mathematical formulation for the control problem of maximizing reliability of averaged system (28). Both the optimal control law and the reliability function of optimally control system (28) can be obtained from solving these equations.

The control problem of maximizing mean first-passage time can be formulated similarly. Let $E[\tau(\bar{A}, \bar{\Gamma}, u)]$ denote the mean first-passage time of controlled system (28). Define the value function

$$
V_{1}(\bar{a}, \bar{\gamma})=\sup _{u \in U_{c}} E[\tau(\bar{A}, \bar{\Gamma}, u)],
$$

which implies $V_{1}$ is the mean first-passage time of optimally controlled system (28). Based on the dynamical programming principle [21], the following dynamical programming equation for value function $V_{1}$ can be derived from Eq. (28):

$$
\begin{gathered}
\sup _{u \in U} \varepsilon\left\{\left[\bar{m}_{1}^{(1)}+\left\langle F_{1}^{(2)}\right\rangle_{\varphi}\right] \frac{\partial}{\partial \bar{a}}+\left[\bar{m}_{2}^{(1)}-\frac{q}{p}\left\langle F_{2}^{(2)}\right\rangle_{\varphi}\right] \frac{\partial}{\partial \bar{\gamma}}+\frac{1}{2} \bar{b}_{11} \frac{\partial^{2}}{\partial \bar{a}^{2}}\right. \\
\left.+\frac{1}{2} \bar{b}_{22} \frac{\partial^{2}}{\partial \bar{\gamma}^{2}}+\bar{b}_{12} \frac{\partial^{2}}{\partial \bar{a} \partial \bar{\gamma}}\right\} V_{1}(\bar{a}, \bar{\gamma})=-1, \quad \bar{a}, \bar{\gamma} \in \Omega_{s} .
\end{gathered}
$$

The boundary conditions associated with Eq. (38) are

$$
\begin{gathered}
V_{1}\left(\bar{a}_{c}, \bar{\gamma}\right)=0, \\
V_{1}(0, \bar{\gamma})=\text { finite, } \\
V(\bar{a}, \bar{\gamma}+2 n \pi)=V(\bar{a}, \bar{\gamma}) .
\end{gathered}
$$

Eqs. (38)-(41) are the mathematical formulation for the control problem of maximizing mean first-passage time. Solving Eqs. (38)-(41) yields both optimal control law and the mean firstpassage time of optimally controlled system (28).

Note that boundary conditions (34) and (40) implied that $\bar{A}(t)$ should not cross boundary $\bar{a}=0$. They are qualitative and can be made to be quantitative by using Eqs. (32) and (38) and by examining the behaviors of the drift and diffusion coefficients of Eqs. (32) and (38) at boundary $\bar{a}=0$. It will be illustrated in the following example.

The optimal control law can be determined from maximizing the left side of Eq. (32) or (38) with respect to $u \in U_{c}$. Since $V$ is a periodic function of $\bar{\gamma}$, the left side of Eq. (32) will be maximum when the term $\left\langle F_{1}^{(2)}\right\rangle_{\varphi} \partial V / \partial \bar{a}$ reaches maximum. Suppose that the control constraint $u \in U_{c}$ is of the form

$$
|u| \leqslant u_{0},
$$

where $u_{0}$ is a positive constant. Then

$$
\left\langle F_{1}^{(2)}\right\rangle_{\varphi} \frac{\partial V}{\partial \bar{a}}=\left\langle-\frac{u}{g(a+b)(1+h)} a v(a, \varphi) \sin \varphi\right\rangle_{\varphi} \frac{\partial V}{\partial \bar{a}},
$$


will be maximum when $|u|=u_{0}$ and $(-u A v(a, \varphi) \sin \varphi \partial V / \partial a) /[g(a+b)(1+h)]$ is positive, i.e.,

$$
u^{*}=u_{0} \operatorname{sgn}\left[-a v(a, \varphi) \sin \varphi \frac{\partial V}{\partial \bar{a}} / g(a+b)(1+h)\right] \text {. }
$$

Usually, $g(a+b)(1+h)>0$ and $\partial V / \partial \bar{a}<0$ since reliability is a monotonously decreasing function of $\bar{a}$ (e.g., see Fig. 5). Thus, Eq. (44) is reduced to

$$
u^{*}=-u_{0} \operatorname{sgn}(-a v(a, \varphi) \sin \varphi)=-u_{0} \operatorname{sgn}(\dot{X}(t))
$$

Eq. (45) implies that optimal control is a bang-bang control. $u^{*}$ has a constant magnitude $u_{0}$. It is in the opposite direction of $\dot{X}(t)$ and changes its direction at $\dot{X}(t)=0$.

Inserting Eq. (45) into Eq. (32) to replace $u$ and averaging $F_{i}^{(2)}$, one obtains the final dynamical programming equation for the control problem of maximizing reliability

$$
\left[\frac{\partial}{\partial t}+\bar{m}_{1} \frac{\partial}{\partial \bar{a}}+\bar{m}_{2} \frac{\partial}{\partial \bar{\gamma}}+\frac{1}{2} \bar{b}_{11} \frac{\partial^{2}}{\partial \bar{a}^{2}}+\frac{1}{2} \bar{b}_{22} \frac{\partial^{2}}{\partial \bar{\gamma}^{2}}+\bar{b}_{12} \frac{\partial^{2}}{\partial \bar{a} \partial \bar{\gamma}}\right] V(t, \bar{a}, \bar{\gamma})=0, \quad \bar{a}, \bar{\gamma} \in \Omega_{s}, \quad 0 \leqslant t \leqslant t_{f},
$$

where

$$
\begin{aligned}
& \bar{m}_{1}(\bar{a}, \bar{\gamma})=\bar{m}_{1}^{(1)}+\left\langle\frac{-u^{*} a v(a, \varphi) \sin \varphi}{g(a+b)(1+h)}\right\rangle_{\varphi}, \\
& \bar{m}_{2}(\bar{a}, \bar{\gamma})=\bar{m}_{2}^{(1)}+\frac{q}{p}\left\langle\frac{u^{*} v(a, \varphi)(\cos \varphi+h}{g(a+b)(1+h)}\right\rangle_{\varphi} .
\end{aligned}
$$

The boundary and final time conditions are still those in Eqs. (33)-(36).

Similarly, the optimal control and the final dynamical programming equation for the control problem of maximizing mean first-passage time are Eq. (45) and

$$
\varepsilon\left\{\bar{m}_{1} \frac{\partial}{\partial \bar{a}}+\bar{m}_{2} \frac{\partial}{\partial \bar{\gamma}}+\frac{1}{2} \bar{b}_{11} \frac{\partial^{2}}{\partial \bar{a}^{2}}+\frac{1}{2} \bar{b}_{22} \frac{\partial^{2}}{\partial \bar{\gamma}^{2}}+\bar{b}_{12} \frac{\partial^{2}}{\partial \bar{a} \partial \bar{\gamma}}\right\} V_{1}(\bar{a}, \bar{\gamma})=-1,
$$

respectively. The boundary conditions are still those in Eqs. (39)-(41).

Inserting Eq. (47) into Eq. (28) to replace its drift coefficients yields the completely averaged Itô equations for $\bar{A}$ and $\bar{\Gamma}$

$$
\begin{aligned}
& \mathrm{d} \bar{A}=\varepsilon \bar{m}_{1}(\bar{A}, \bar{\Gamma}) \mathrm{d} t+\varepsilon^{1 / 2} \bar{\sigma}_{1 r}(\bar{A}) \mathrm{d} B_{r}(t), \\
& \mathrm{d} \bar{\Gamma}=\varepsilon \bar{m}_{2}(\bar{A}, \bar{\Gamma}) \mathrm{d} t+\varepsilon^{1 / 2} \bar{\sigma}_{2 r}(\bar{A}) \mathrm{d} B_{r}(t) .
\end{aligned}
$$

\section{Backward Kolmogorov equation and Pontryagin equation of optimally controlled system}

Eq. (49) is the completely averaged Itô equations of optimally controlled system (28). The conditional reliability function of system (49) is defined by

$$
R_{o p t}\left(t_{1} \mid \bar{a}_{0}, \bar{\gamma}_{0}\right)=P\left\{\left[\bar{A}\left(\tau, u^{*}\right), \bar{\Gamma}\left(\tau, u^{*}\right)\right] \in \Omega_{s}, \quad \tau \in\left[0, t_{1}\right] \mid\left(\bar{a}_{0}, \bar{\gamma}_{0}\right) \in \Omega_{s}\right\} .
$$


Since $\bar{A}, \bar{\Gamma}$ is a homogeneous diffusion process, $R_{o p t}$ is governed by the following backward Kolmogorov equation [22]:

$$
\left[-\frac{\partial}{\partial t_{1}}+\bar{m}_{1} \frac{\partial}{\partial \bar{a}_{0}}+\bar{m}_{2} \frac{\partial}{\partial \bar{\gamma}_{0}}+\frac{1}{2} \bar{b}_{11} \frac{\partial^{2}}{\partial \bar{a}_{0}^{2}}+\frac{1}{2} \bar{b}_{22} \frac{\partial^{2}}{\partial \bar{\gamma}_{0}^{2}}+\bar{b}_{12} \frac{\partial^{2}}{\partial \bar{a}_{0} \partial \bar{\gamma}_{0}}\right] R_{o p t}=0, \quad \bar{a}_{0}, \bar{\gamma}_{0} \in \Omega_{s},
$$

with boundary conditions

$$
\begin{gathered}
R_{\text {opt }}\left(t_{1} \mid \bar{a}_{c}, \bar{\gamma}_{0}\right)=0, \\
R_{\text {opt }}\left(t_{1} \mid 0, \bar{\gamma}_{0}\right)=\text { finite, } \\
R_{\text {opt }}\left(t_{1} \mid \bar{a}_{0}, \bar{\gamma}_{0}+2 n \pi\right)=R_{\text {opt }}\left(t_{1} \mid \bar{a}_{0}, \bar{\gamma}_{0}\right),
\end{gathered}
$$

and initial condition

$$
R_{\text {opt }}\left(0 \mid \bar{a}_{0}, \bar{\gamma}_{0}\right)=1, \quad \bar{a}_{0}, \bar{\gamma}_{0} \in \Omega,
$$

where $\bar{m}_{i}=\bar{m}_{i}\left(a_{0}, \gamma_{0}\right), \bar{b}_{i j}=\bar{b}_{i j}\left(a_{0}, \gamma_{0}\right)$ are defined by Eqs. (29) and (47) with $\bar{a}, \bar{\gamma}$ replaced by $\bar{a}_{0}, \bar{\gamma}_{0}$.

A comparison between Eqs. (31) and (50) reveals that

$$
R_{\text {opt }}\left(t_{f} \mid \bar{a}_{0}, \bar{\gamma}_{0}\right)=V\left(0, \bar{a}_{0}, \bar{\gamma}_{0}\right) \text {. }
$$

Note that in Eq. (51) $t_{1}$ is a forward time running from 0 to $t_{f}$ while in Eq. (46) $t$ is a backward time running from $t_{f}$ to 0 . Introducing transformations

$$
\begin{aligned}
& t_{1}=t_{f}-t, \\
& V(t, \bar{a}, \bar{\gamma}) \rightarrow R_{o p t}\left(t_{1} \mid \bar{a}_{0}, \bar{\gamma}_{0}\right),
\end{aligned}
$$

Eq. (46) will be of the same form of Eq. (51) and final time condition (36) becomes initial condition (55) while boundary conditions (33)-(35) becomes (52)-(54).

The conditional probability of first-passage failure of optimally controlled system is

$$
P_{\text {opt }}\left(t_{1} \mid \bar{a}_{0}, \bar{\gamma}_{0}\right)=1-R_{\text {opt }}\left(t_{1} \mid \bar{a}_{0}, \bar{\gamma}_{0}\right) \text {. }
$$

The conditional probability density of first-passage time $T$ is then

$$
p_{\text {opt }}\left(T \mid \bar{a}_{0}, \bar{\gamma}_{0}\right)=\left.\frac{\partial P_{o p t}\left(t_{1} \mid \bar{a}_{0}, \bar{\gamma}_{0}\right)}{\partial t_{1}}\right|_{t_{1}=T}=-\left.\frac{\partial R_{o p t}\left(t_{1} \mid \bar{a}_{0}, \bar{\gamma}_{0}\right)}{\partial t_{1}}\right|_{t_{1}=T} .
$$

The mean first-passage time is defined by

$$
\mu_{1, \text { opt }}\left(\bar{a}_{0}, \bar{\gamma}_{0}\right)=\int_{0}^{\infty} T p_{\text {opt }}\left(T \mid \bar{a}_{0}, \bar{\gamma}_{0}\right) \mathrm{d} T=\int_{0}^{\infty} R_{\text {opt }}\left(T \mid \bar{a}_{0}, \bar{\gamma}_{0}\right) \mathrm{d} T .
$$

The following Pontryagin equation for the conditional mean first-passage time of optimally controlled system can be derived from Eqs. (51) and (60):

$$
\varepsilon\left[\bar{m}_{1} \frac{\partial}{\partial \bar{a}_{0}}+\bar{m}_{2} \frac{\partial}{\partial \bar{\gamma}_{0}}+\frac{1}{2} \bar{b}_{11} \frac{\partial^{2}}{\partial \bar{a}_{0}^{2}}+\frac{1}{2} \bar{b}_{22} \frac{\partial^{2}}{\partial \bar{\gamma}_{0}^{2}}+\bar{b}_{12} \frac{\partial^{2}}{\partial \bar{a}_{0} \partial \bar{\gamma}_{0}}\right] \mu_{1, o p t}=-1 .
$$

The boundary conditions associated with Eq. (61) are

$$
\begin{gathered}
\mu_{1, \text { opt }}\left(\bar{a}_{c}, \bar{\gamma}_{0}\right)=0, \\
\mu_{1, \text { opt }}\left(0, \bar{\gamma}_{0}\right)=\text { finite, }
\end{gathered}
$$




$$
\mu_{1, \text { opt }}\left(\bar{a}_{0}, \bar{\gamma}_{0}+2 n \pi\right)=\mu_{1, \text { opt }}\left(\bar{a}_{0}, \bar{\gamma}_{0}\right) .
$$

Obviously, dynamical programming equation (48) and its boundary conditions (39)-(41) are of the same form of Eqs. (61)-(64). Thus, we have

$$
\mu_{1, o p t}\left(\bar{a}_{0}, \bar{\gamma}_{0}\right)=V_{1}(\bar{a}, \bar{\gamma})_{\bar{a}=\bar{a}_{0}, \bar{\gamma}=\overline{\gamma_{0}}} .
$$

Therefore, we can first solve dynamical programming equation (46) together with boundary conditions (33)-(35) and final time condition (36) to obtain $V(t, \bar{a}, \bar{\gamma})$ and then obtain the conditional reliability function and conditional probability density of first-passage time of optimally controlled system by using Eqs. (57) and (59), respectively. Or, we can first transform dynamical programming equation (46) into backward Kolmogorov equation (51) by using transformation (57) and then solve Eq. (51) together with boundary and initial conditional (52)-(55) to obtain the conditional reliability function, and finally obtain the conditional probability density of first-passage time of optimally controlled system by using Eq. (59). As for the conditional mean first-passage time of optimally controlled system, we can either first solve dynamical programming equation (48) together with boundary conditions (39)-(41) to obtain value function $V_{1}(\bar{a}, \bar{\gamma})$ and obtain it by using Eq. (65), or first transform dynamical programming equation (48) into Pontryagin equation (61) and then solve Eq. (61) together with boundary conditions (62)-(64) to obtain it. It is also possible to obtain the conditional mean first-passage time of optimally controlled system from the conditional probability density of first-passage time or conditional reliability function by using Eq. (60).

Finally, it is noted that the control law (45) is optimal for the averaged system (28). For original system (1), it is only quasi-optimal or nearly optimal. To simplify the statement, it is simply called optimal bounded control for system (1).

\section{Example}

Consider the optimal bounded control of a Duffing oscillator subject to external harmonic excitation and external and parametric white-noise excitations. The motion equation of the system is of the form

$$
\ddot{X}+\omega^{2} X+\alpha X^{3}=-\beta \dot{X}+E \cos \Omega t+\xi_{1}(t)+X \xi_{2}(t)+u,
$$

where $\omega, \alpha, \beta, E, \Omega$ are positive constants denoting the natural frequency of degenerate linear oscillator, intensity of non-linearity, damping coefficient, amplitude and frequency of harmonic excitation, respectively; $\xi_{k}(t)(k=1,2)$ are independent Gaussian white noises in the sense of Stratonovich with intensities $2 D_{k}$. It is assumed that $\beta, E$ and $D_{k}$ are of the same order of $\varepsilon$.

For this oscillator,

$$
\begin{aligned}
& U(x)=\omega^{2} x^{2} / 2+\alpha x^{4} / 4, \\
& g(x)=\mathrm{d} U / \mathrm{d} x=\omega^{2} x+\alpha x^{3},
\end{aligned}
$$


and

$$
\begin{aligned}
& v^{-1}(a, \varphi)=\left[\left(\omega^{2}+3 \alpha a^{2} / 4\right)(1+\lambda \cos 2 \varphi)\right]^{-1 / 2}=\sum_{n=0}^{\infty} C_{2 n}(a) \cos 2 n \varphi \\
& C_{2 n}(a)=\frac{1}{2 \pi} \int_{0}^{2 \pi} v^{-1}(a, \varphi) \cos 2 n \varphi \mathrm{d} \varphi \\
& \lambda=\alpha a^{2} / 4\left(\omega^{2}+3 \alpha a^{2} / 4\right) .
\end{aligned}
$$

Two cases are considered in the following.

Case 1: Primary external resonance: In this case $p=q=1$ and

$$
\Omega / \omega(a)=1+\sigma,
$$

where $\sigma$ is of the same order of $\varepsilon$. By using the generalized van der Pol transformations (15) with $B=0$, Eq. (66) is converted into

$$
\begin{aligned}
\frac{\mathrm{d} A}{\mathrm{~d} t} & =F_{1}^{(1)}(A, \Phi, \Omega t)+F_{1}^{(2)}(A, \Phi, u)+h_{11}(A, \Phi) \xi_{1}(t)+h_{12}(A, \Phi) \xi_{2}(t), \\
\frac{\mathrm{d} \Theta}{\mathrm{d} t} & =F_{2}^{(1)}(A, \Phi, \Omega t)+F_{2}^{(2)}(A, \Phi, u)+h_{21}(A, \Phi) \xi_{1}(t)+h_{22}(A, \Phi) \xi_{2}(t),
\end{aligned}
$$

where

$$
\begin{aligned}
F_{1}^{(1)} & =-\frac{A}{g(A)}[\beta A v(A, \Phi) \sin \Phi+E \cos \Omega t] v(A, \Phi) \sin \Phi, \\
F_{1}^{(2)} & =-\frac{u}{g(A)} A v(A, \Phi) \sin \Phi, \\
F_{2}^{(1)} & =-\frac{1}{g(A)}[\beta A v(A, \Phi) \sin \Phi+E \cos \Omega t] v(A, \Phi) \cos \Phi, \\
F_{2}^{(2)} & =-\frac{u}{g(A)} v(A, \Phi) \cos \Phi, \\
h_{11} & =-\frac{A}{g(A)} v(A, \Phi) \sin \Phi, \quad h_{12}=-\frac{A^{2}}{g(A)} v(A, \Phi) \sin \Phi \cos \Phi, \\
h_{21} & =-\frac{1}{g(A)} v(A, \Phi) \cos \Phi, \quad h_{22}=-\frac{A}{g(A)} v(A, \Phi) \cos ^{2} \Phi .
\end{aligned}
$$

Eq. (70) can be modelled as the following Itô stochastic differential equations by adding WongZakai correction terms:

$$
\begin{aligned}
\mathrm{d} A & =\left[m_{1}^{(1)}(A, \Phi, \Omega t)+F_{1}^{(2)}(A, \Phi, u)\right] \mathrm{d} t+\sigma_{1 k}(A, \Phi) \mathrm{d} B_{k}(t), \\
\mathrm{d} \Theta & =\left[m_{2}^{(1)}(A, \Phi, \Omega t)+F_{2}^{(2)}(A, \Phi, u)\right] \mathrm{d} t+\sigma_{2 k}(A, \Phi) \mathrm{d} B_{k}(t), \quad k=1,2,
\end{aligned}
$$

where

$$
\begin{aligned}
& m_{i}^{(1)}=F_{i}^{(1)}+D_{k}\left(h_{1 k} \frac{\partial h_{i k}}{\partial A}+h_{2 k} \frac{\partial h_{i k}}{\partial \Phi}\right), \\
& b_{i j}=\sigma_{i r} \sigma_{j r}=2 D_{k} h_{i k} h_{j k}, \quad i, j, k=1,2 .
\end{aligned}
$$


Introducing new variable

$$
\Gamma=\sigma \tau-\Theta
$$

Eq. (72) is transformed into

$$
\begin{aligned}
& \mathrm{d} A=\left[m_{1}^{(1)}(A, \Phi, \Psi+\Gamma)+F_{1}^{(2)}(A, \Phi, u)\right] \mathrm{d} t+\sigma_{1 k}(A, \Phi) \mathrm{d} B_{k}(t), \\
& \mathrm{d} \Gamma=\left[m_{2}^{(1)}(A, \Phi, \Psi+\Gamma)-F_{2}^{(2)}(A, \Phi, u)+(\Omega / \omega(A)-1) v(A, \Phi)\right] \mathrm{d} t-\sigma_{2 k}(A, \Phi) \mathrm{d} B_{k}(t) .
\end{aligned}
$$

Averaging the drift and diffusion coefficients in Itô equation (75) with respect to $\Phi$ leads to

$$
\begin{aligned}
\mathrm{d} \bar{A} & =\left[\bar{m}_{1}^{(1)}(\bar{A}, \bar{\Gamma})+\left\langle F_{1}^{(2)}(A, \Phi, u)\right\rangle_{\Phi}\right] \mathrm{d} t+\bar{\sigma}_{1 k}(\bar{A}) \mathrm{d} B_{k}(t), \\
\mathrm{d} \bar{\Gamma} & =\left[\bar{m}_{2}^{(1)}(\bar{A}, \bar{\Gamma})-\left\langle F_{2}^{(2)}(A, \Phi, u)\right\rangle_{\Phi}\right] \mathrm{d} t+\bar{\sigma}_{2 k}(\bar{A}) \mathrm{d} B_{k}(t),
\end{aligned}
$$

where

$$
\begin{aligned}
& \bar{m}_{1}^{(1)}(\bar{A}, \bar{\Gamma})=-\beta \bar{A}\left(\omega^{2}+5 \alpha \bar{A}^{2} / 8\right) / 2\left(\omega^{2}+\alpha \bar{A}^{2}\right)+E \sin \bar{\Gamma}\langle v(\bar{A}, \Phi) \sin \Phi \\
&\left.\times \sin \left(\Phi+\Omega \sum_{n=1}^{\infty} \frac{1}{n} C_{n}(\bar{A}) \sin n \Phi\right)\right\rangle_{\Phi} /\left(\omega^{2}+\alpha \bar{A}^{2}\right) \\
&-\alpha D_{1} \bar{A}\left(3 \omega^{2}+3 \alpha \bar{A}^{2} / 2\right) / 4\left(\omega^{2}+\alpha \bar{A}^{2}\right)^{3}+D_{1}\left(\omega^{2}+7 \alpha \bar{A}^{2} / 8\right) / 2 \bar{A}\left(\omega^{2}+\alpha \bar{A}^{2}\right)^{2} \\
&+D_{2} \omega^{2} \bar{A}\left(\omega^{2}+\alpha \bar{A}^{2} / 2\right) / 8\left(\omega^{2}+\alpha \bar{A}^{2}\right)^{3}+D_{2} \bar{A}\left(\omega^{2}+7 \alpha \bar{A}^{2} / 8\right) / 4\left(\omega^{2}+\alpha \bar{A}^{2}\right)^{2}, \\
& \bar{m}_{2}^{(1)}(\bar{A}, \bar{\Gamma})= E \cos \bar{\Gamma}\left\langle v(\bar{A}, \Phi) \cos \Phi \cos \left(\Phi+\Omega \sum_{n=1}^{\infty} \frac{1}{n} C_{n}(\bar{A}) \sin n \Phi\right)\right\rangle_{\Phi} / \bar{A}\left(\omega^{2}+\alpha \bar{A}^{2}\right) \\
&+\left[\Omega C_{0}(\bar{A})-1\right]\langle v(\bar{A}, \Phi)\rangle_{\Phi}, \\
& \bar{b}_{11}(\bar{A})= \bar{\sigma}_{1 k} \bar{\sigma}_{1 k} \\
&= D_{1}\left(\omega^{2}+5 \alpha \bar{A}^{2} / 8\right) /\left(\omega^{2}+\alpha \bar{A}^{2}\right)^{2}+D_{2} A^{2}\left(\omega^{2}+3 \alpha \bar{A}^{2} / 4\right) / 4\left(\omega^{2}+\alpha \bar{A}^{2}\right)^{2}, \\
& \bar{b}_{22}(\bar{A})= \bar{\sigma}_{2 k} \bar{\sigma}_{2 k} \\
&= D_{1}\left(\omega^{2}+7 \alpha \bar{A}^{2} / 8\right) / \bar{A}^{2}\left(\omega^{2}+\alpha \bar{A}^{2}\right)^{2}+2 D_{2}\left(3 \omega^{2} / 8+11 \alpha \bar{A}^{2} / 32\right) /\left(\omega^{2}+\alpha \bar{A}^{2}\right)^{2}, \\
& \bar{b}_{12}(\bar{A})=\bar{b}_{21}(\bar{A})=\bar{\sigma}_{1 k} \bar{\sigma}_{2 k}=0 .
\end{aligned}
$$

The dynamical programming equation for the control problem of maximizing reliability is of the form of Eq. (32) with $\bar{m}_{i}^{(1)}, \bar{b}_{i j}$ defined by Eq. (77). Suppose that the control constraint is of the form of Eq. (42). Then the optimal control is of the form of Eq. (45). Inserting $u^{*}$ in Eq. (45) into $F_{i}^{(2)}$ in Eq. (71) and then averaging $F_{i}^{(2)}$ with respect $\Phi$, one obtains

$$
\begin{aligned}
& \left\langle F_{1}^{(2)}\left(A, \Phi, u^{*}\right)\right\rangle_{\Phi}=\frac{-A}{g(A+B)(1+h)}\left\langle u^{*} v(A, \Phi) \sin \Phi\right\rangle_{\Phi}, \\
& \left\langle F_{2}^{(2)}\left(A, \Phi, u^{*}\right)\right\rangle_{\Phi}=\frac{-1}{g(A+B)(1+h)}\left\langle u^{*} v(A, \Phi) \cos \Phi\right\rangle_{\Phi} .
\end{aligned}
$$


The conditional reliability function of optimally controlled system (66) can be obtained from solving backward Kolmogorov equation (51) with $\bar{b}_{i j}$ defined by Eq. (77) and

$$
\begin{aligned}
& \bar{m}_{1}=\bar{m}_{1}^{(1)}\left(\bar{a}_{0}, \bar{\gamma}_{0}\right)+\left.\left\langle F_{1}^{(2)}\left(A, \Phi, u^{*}\right)\right\rangle_{\Phi}\right|_{A=a_{0}}, \\
& \bar{m}_{2}=\bar{m}_{2}^{(1)}\left(\bar{a}_{0}, \bar{\gamma}_{0}\right)-\left.\left\langle F_{2}^{(2)}\left(A, \Phi, u^{*}\right)\right\rangle_{\Phi}\right|_{A=a_{0}},
\end{aligned}
$$

where $\bar{m}_{i}^{(1)}$ defined by Eq. (77) and $\left\langle F_{i}^{(2)}\right\rangle$ defined by Eq. (78). The associated two boundary conditions are Eqs. (52) and (54) and initial condition is Eq. (55). One more boundary condition, Eq. (53) can be replaced by Eq. (51) with $\bar{m}_{i}$ and $\bar{b}_{i j}$ at $\bar{a}_{0}=0$. The backward Kolmogorov equation can be solved by using finite difference method with modified standard Thomas algorithm. Then the conditional probability density of first-passage time of optimally controlled system (66) can be obtained from the conditional reliability function by using Eq. (59). The mean first-passage time of optimally controlled system (66) can be obtained either from the conditional reliability function by using Eq. (60) or from solving Pontryagin equation (61) together with boundary conditions (62)-(64), where boundary Eq. (63) is replaced by Eq. (61) with $\bar{m}_{i}, \bar{b}_{i j}$ defined by Eqs. (77) and (79) at $\bar{a}_{0}=0$.

Some numerical results are shown in Figs. 2-4, where solid line denotes analytical results while - 14 the results from digital simulation of original system (66). It is seen that the analytical results are in rather good agreement with those from digital simulation and the reliability of the system improved greatly by feedback control. The reliability as function of $\bar{a}_{0}$ at fixed $t$ and $\bar{\gamma}_{0}$ is shown in Fig. 5. It is shown that reliability is a monotonously decreasing function of $\bar{a}_{0}$, which justifies the derivation from Eq. (44) to Eq. (45).

Case 2: Primary parametric resonance: In this case $p=1, q=2$ and

$$
\frac{\Omega}{\omega(a)}=2+\sigma,
$$

where $\sigma$ is of order of $\varepsilon$. Introduce new variable

$$
\Gamma=\sigma \tau-2 \Theta .
$$

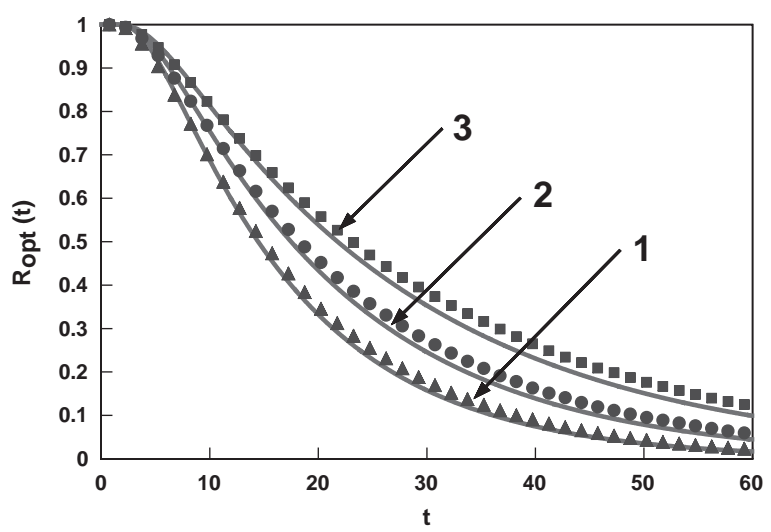

Fig. 2. Reliability function of optimally controlled system (66) in primary external resonance for given initial condition: $\omega=1.0, \Omega=1.0, \alpha=0.6, \beta=0.01, E=0.01, D_{1}=0.03, D_{2}=0.04, \bar{a}_{0}=0.24, \bar{\gamma}_{0}=0.1257,1-u_{0}=0.0 ; 2-u_{0}=$ $0.02 ; 3-u_{0}=0.04$. - , analytical result; $\boldsymbol{\square} \bullet \mathbf{\Delta}$, result from digital simulation. 


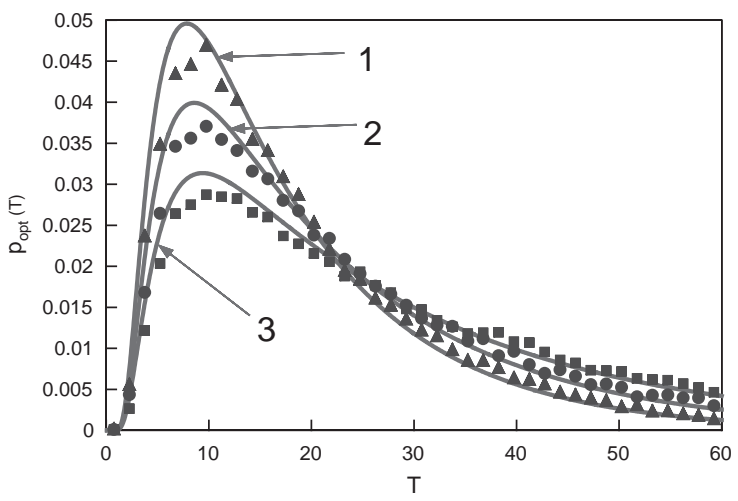

Fig. 3. Probability density of first-passage time of optimally controlled system (66) in primary external resonance for given initial condition. The parameters are the same as those in Fig. 2. _ _ , analytical result; $\square \mathbf{\square}$, result from digital simulation.

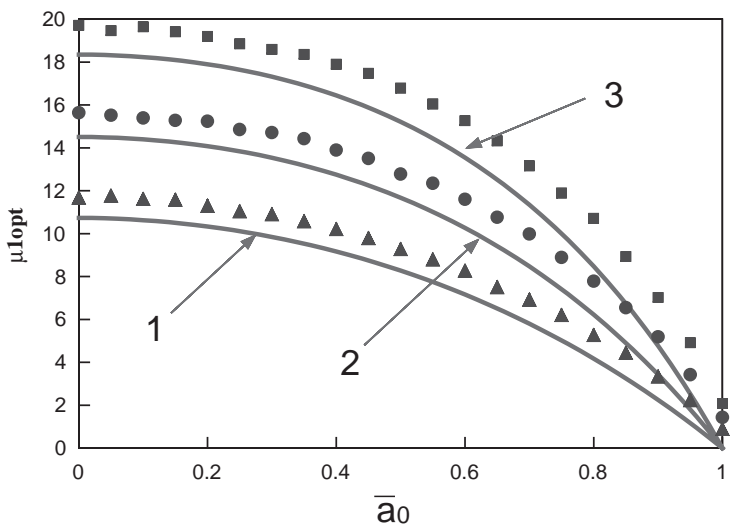

Fig. 4. Mean first-passage time of optimally controlled system (66) in primary external resonance: $\omega=1.2, \Omega=$ $1.2, \alpha=0.7, \beta=0.01, E=0.01, D_{1}=0.08, D_{2}=0.04, \bar{\gamma}_{0}=2.514,1-u_{0}=0 ; 2-u_{0}=0.06 ; 3-u_{0}=0.1 .-$, analytical result; $\mathbf{\square} \boldsymbol{\Lambda}$, result from digital simulation.

Applying the stochastic averaging method to Eq. (66) leads equations similar to Eq. (76), where

$$
\begin{aligned}
\bar{m}_{1}^{(1)}(\bar{A}, \bar{\Gamma})= & -\beta \bar{A}\left(\omega^{2}+5 \alpha \bar{A}^{2} / 8\right) / 2\left(\omega^{2}+\alpha \bar{A}^{2}\right) \\
& +E \sin \bar{\Gamma}\left\langle v(\bar{A}, \Phi) \sin \Phi \sin \left(2 \Phi+\Omega \sum_{n=1}^{\infty} \frac{1}{n} C_{n}(\bar{A}) \sin n \Phi\right)\right\rangle_{\Phi} /\left(\omega^{2}+\alpha \bar{A}^{2}\right) \\
& -\alpha D_{1} \bar{A}\left(3 \omega^{2}+3 \alpha \bar{A}^{2} / 2\right) / 4\left(\omega^{2}+\alpha \bar{A}^{2}\right)^{3}+D_{1}\left(\omega^{2}+7 \alpha \bar{A}^{2} / 8\right) / 2 \bar{A}\left(\omega^{2}+\alpha \bar{A}^{2}\right)^{2} \\
& +D_{2} \omega^{2} \bar{A}\left(\omega^{2}+\alpha \bar{A}^{2} / 2\right) / 8\left(\omega^{2}+\alpha \bar{A}^{2}\right)^{3}+D_{2} \bar{A}\left(\omega^{2}+7 \alpha \bar{A}^{2} / 8\right) / 4\left(\omega^{2}+\alpha \bar{A}^{2}\right)^{2}, \\
\bar{m}_{2}^{(1)}(\bar{A}, \bar{\Gamma})= & E \cos \bar{\Gamma}\left\langle v(\bar{A}, \Phi) \cos \Phi \cos \left(2 \Phi+\Omega \sum_{n=1}^{\infty} \frac{1}{n} C_{n}(\bar{A}) \sin n \Phi\right)\right\rangle_{\Phi} / \bar{A}\left(\omega^{2}+\alpha \bar{A}^{2}\right) \\
& +\left[\Omega C_{0}(\bar{A})-2\right]\langle v(\bar{A}, \Phi)\rangle_{\Phi},
\end{aligned}
$$




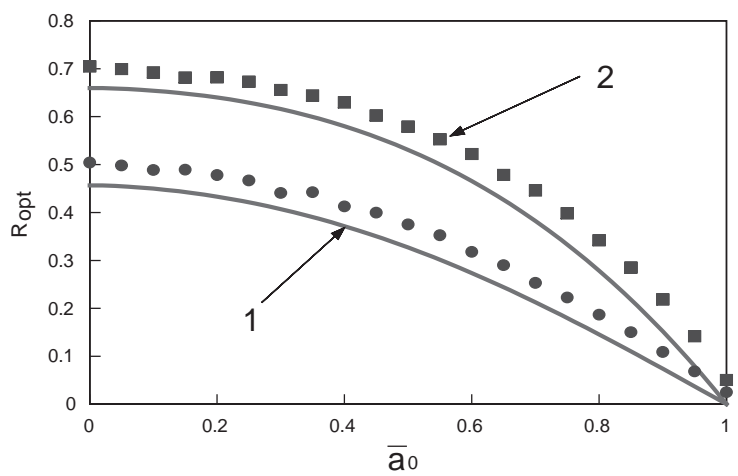

Fig. 5. Reliability of optimally controlled system (66) in primary external resonance as function of initial displacement amplitude $\bar{a}_{0}: \omega=1.0, \Omega=1.0, \alpha=0.6, \beta=0.01, E=0.01, D_{1}=0.03, D_{2}=0.04, \bar{\gamma}_{0}=2.514, t=20, \quad 1-u_{0}=$ $0.02 ; 2-u_{0}=0.06$. - , analytical result; $\mathbf{\square}$, result from digital simulation.

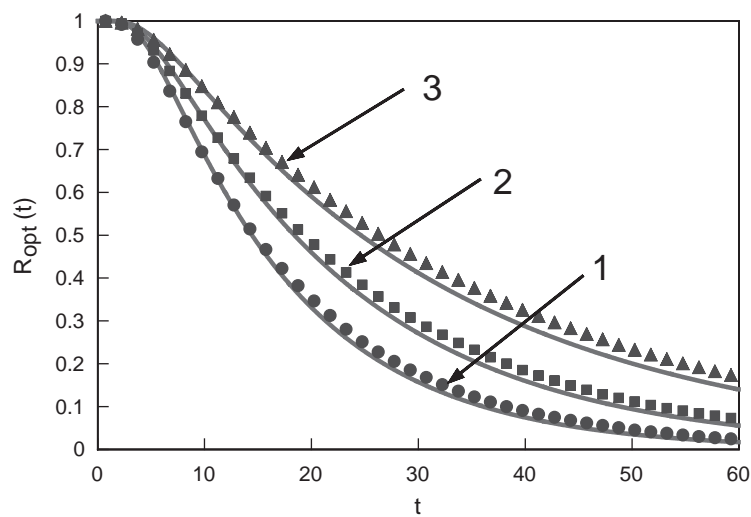

Fig. 6. Reliability function of optimally controlled system (66) in primary parametric resonance for given initial condition: $\omega=1.0, \Omega=2.0, \alpha=0.6, \beta=0.01, E=0.01, D_{1}=0.03, D_{2}=0.04, \bar{a}_{0}=0.24, \overline{\gamma_{0}}=1.257,1-u_{0}=0$; $2-u_{0}=0.025 ; 3-u_{0}=0.05$. — , analytical result; $\mathbf{\square} \bullet \mathbf{\Lambda}$, result from digital simulation.

$$
\begin{gathered}
\bar{b}_{11}(\bar{A})=\bar{\sigma}_{1 r} \bar{\sigma}_{1 r} \\
=D_{1}\left(\omega^{2}+5 \alpha \bar{A}^{2} / 8\right) /\left(\omega^{2}+\alpha \bar{A}^{2}\right)^{2}+D_{2} \bar{A}^{2}\left(\omega^{2}+3 \alpha \bar{A}^{2} / 4\right) / 4\left(\omega^{2}+\alpha \bar{A}^{2}\right)^{2}, \\
\bar{b}_{22}(\bar{A})=\bar{\sigma}_{2 r} \bar{\sigma}_{2 r} \\
=4 D_{1}\left(\omega^{2}+7 \alpha \bar{A}^{2} / 8\right) / \bar{A}^{2}\left(\omega^{2}+\alpha \bar{A}^{2}\right)^{2}+8 D_{2}\left(3 \omega^{2} / 8+11 \alpha \bar{A}^{2} / 32\right) /\left(\omega^{2}+\alpha \bar{A}^{2}\right)^{2}, \\
\bar{b}_{12}(\bar{A})=\bar{b}_{21}(\bar{A})=\bar{\sigma}_{1 r} \bar{\sigma}_{2 r}=0 .
\end{gathered}
$$

Then, following the same procedure as that in case 1, one obtains the conditional reliability function, conditional probability density and mean of first-passage time of optimally controlled system (66).

Some numerical results are shown in Figs. 6-8. It is seen that the agreement between analytical results and those from digital simulation in this case is even better than that in case 1 . 


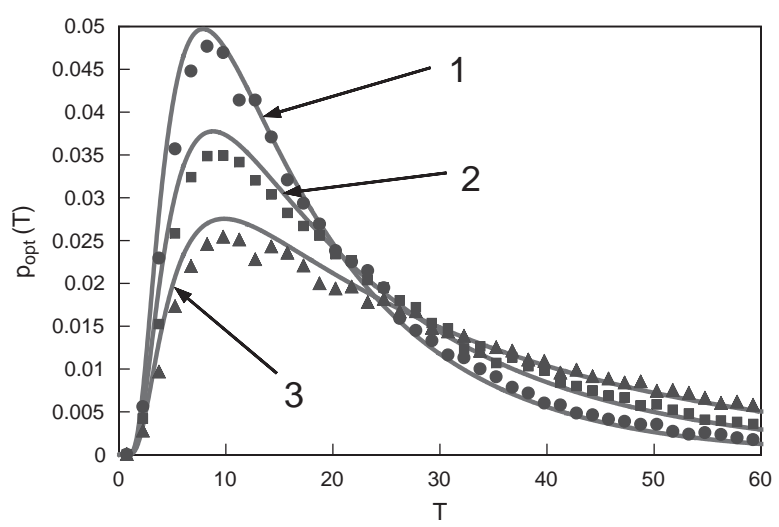

Fig. 7. Probability density of first-passage time of optimally controlled system (66) in primary parametric resonance for given initial condition. The parameters are the same as those in Fig. 6. $\_$, analytical result; $\square \boldsymbol{\bullet}$, result from digital simulation.

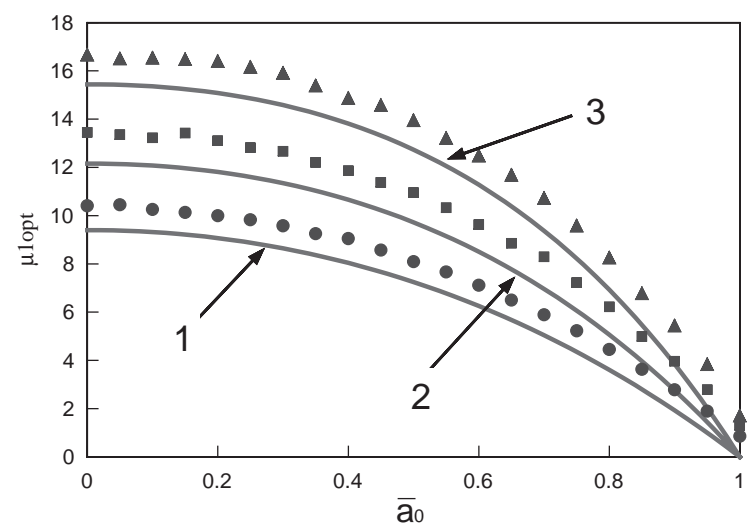

Fig. 8. Mean first-passage time of optimally controlled system (66) in primary parametric resonance: $\omega=1.0, \Omega=$ $2.0, \alpha=0.6, \beta=0.01, E=0.01, D_{1}=0.06, D_{2}=0.08, \bar{\gamma}_{0}=2.514,1-u_{0}=0 ; 2-u_{0}=0.05 ; 3-u_{0}=0.09$. analytical result; $\boldsymbol{\bullet} \boldsymbol{\Lambda}$, result from digital simulation.

\section{Conclusions}

In the present paper a procedure for designing the optimal bounded control of strongly nonlinear systems under combined harmonic and white-noise excitations to minimize the first-passage failure has been proposed for the first time. The procedure consists of applying the stochastic averaging method for strongly non-linear systems under combined harmonic and white-noise excitations, establishing the dynamical programming equations for the control problems of maximizing reliability and of maximizing mean first-passage time based on the averaged Itô equations using dynamical programming principle, determining the optimal control from the 
dynamical programming equations and control constraint, and obtaining the conditional reliability function, conditional probability density and mean of first-passage time of optimally controlled system from solving the associated backward Kolmogorov equation and Pontryagin equation. An example has been worked out in detail to illustrate the application of the proposed procedure. The comparison between the analytical results and those from digital simulation shows that the proposed procedure works quite well. All the results show that the reliability of the system can be greatly improved by the feedback control.

It is noted that the proposed procedure can be easily extended to strongly non-linear systems under combined harmonic and wideband random excitations. This will be the subject of our future research.

\section{Acknowledgements}

The work presented in this paper was supported by the National Natural Science Foundation of China under Grant No. 19972059 and the Special Fund for Doctor Programs in the Institutions of Higher Learning of China under Grant No. 20020335092.

\section{References}

[1] A.H. Nayfeh, S.J. Serhan, Response statistics of non-linear systems to combined deterministic and random excitations, International Journal of Non-Linear Mechanics 25 (1990) 493-509.

[2] R.L. Stratonovich, Topics in the Theory of Random Noise, Vol. 2, Gordon and Breach, New York, 1967.

[3] S.T. Ariaratnam, D.S.F. Tam, Moment Stability of coupled linear systems under combined harmonic and stochastic excitation, in: B.L. Clarkson (Ed.), Stochastic Problems in Dynamics, Pitman, London, 1977, pp. 90-103.

[4] W.Q. Zhu, T.C. Huang, Dynamic instability of liquid free surface in a container with elastic bottom under combined harmonic and stochastic longitudinal excitation, in: T.C. Huang, P.D. Spanos (Eds.), ASME AMD-65, ASME, New York, 1984, pp. 195-222.

[5] M.F. Dimentberg, Statistical Dynamics of Nonlinear and Time-Varying Systems, Research Studies Press Ltd, Wiley, New York, 1988.

[6] N. Sri Namachchivaya, Almost sure stability of dynamical systems under combined harmonic and stochastic excitations, Journal of Sound and Vibration 151 (1991) 77-91.

[7] N.D. Ahn, Random oscillations in a van der Pol system subjected to periodic and random excitations, Soviet Mathematics Doklady 34 (1987) 67-70.

[8] G.Q. Cai, Y.K. Lin, Nonlinear damped systems under simultaneous broad-band and harmonic excitations, Nonlinear Dynamics 6 (1994) 163-177.

[9] Z.L. Huang, W.Q. Zhu, Stochastic averaging of strongly non-linear oscillators under combined harmonic and white noise excitations, Journal of Sound and Vibration 238 (2000) 233-256.

[10] Z. Xu, Y.K. Chueng, Averaging method using generalized harmonic functions for strongly nonlinear oscillators, Journal of Sound and Vibration 174 (1994) 563-576.

[11] G.W. Housner, L.A. Bergman, T.K. Caughey, A.G. Chassiakos, R.O. Clauss, S.F. Masri, R.E. Skelton, T.T. Soong, B.F. Spencer, J.T.P. Yao, Structural control: past, present, and future, Journal of Engineering Mechanics American Society of Civil Engineers 123 (1997) 897-971.

[12] J. Suhardjo, B.F. Spencer Jr., M.K. Sain, Non-linear optimal control of a Duffing system, International Journal of Non-Linear Mechanics 27 (1992) 157-172. 
[13] A.K. Agrawal, J.N. Yang, J.C. Wu, Nonlinear control strategy for Duffing systems, International Journal of NonLinear Mechanics 33 (1998) 829-841.

[14] M. Abdel-Rohman, A.H. Nayfeh, Active control of nonlinear oscillations in bridge, American Society of Civil Engineers, Journal of Engineering Mechanics 113 (1987) 335-348.

[15] P.L. Chow, L. Maestrello, Vibrational control of a non-linear elastic panel, International Journal of Non-Linear Mechanics 36 (2001) 709-718.

[16] J.C. Ji, Local bifurcation control of a forced single-degree-of-freedom nonlinear system: saddle-mode bifurcation, Nonlinear Dynamics 26 (2001) 369-382.

[17] K. Yoshida, A method of optimal control of non-linear stochastic systems with non-quadratic criteria, International Journal of Control 39 (1984) 279-291.

[18] L.G. Crespo, J.Q. Sun, Stochastic optimal control of nonlinear systems via short-time Gaussian approximation and cell mapping, Nonlinear Dynamics 28 (2002) 323-342.

[19] W.Q. Zhu, Z.G. Ying, T.T. Soong, An optimal nonlinear feedback control strategy for randomly excited structural systems, Nonlinear Dynamics 24 (2001) 31-51.

[20] W.Q. Zhu, Z.L. Huang, M.L. Deng, Feedback minimization of first-passage failure of quasi non-integrable Hamiltonian systems, International Journal of Non-Linear Mechanics 37 (2002) 1057-1071.

[21] V.N. Afanas'ev, V.B. Kolmanovskii, V.R. Nosov, Mathematical Theory of Control Systems Design, Kluwer Academic Publishers, Dordrecht, 1996.

[22] W.Q. Zhu, Random Vibration, Science Press, Beijing, 1992 (in Chinese). 